EVS28

KINTEX, Korea, May 3-6, 2015

\title{
Aging of Lithium-Ion Batteries in Electric Vehicles: Impact of Regenerative Braking
}

\author{
Peter Keil ${ }^{1}$, Andreas Jossen ${ }^{1}$ \\ ${ }^{1}$ Institute for Electrical Energy Storage Technology, Technische Universität München, \\ Arcisstr. 21, 80333 Munich, Germany, \\ peter.keil@tum.de
}

\begin{abstract}
In an electric vehicle, energy recovery during regenerative braking causes recharge periods of high current rate, which might damage the Li-ion traction battery. To determine the impact of regenerative braking on battery aging, an experimental cycle life study has been performed: Driving load profiles with different magnitudes of regenerative braking have been applied to high-energy Li-ion cells at different temperatures and states of charge (SoC). An additional calendar life study has enabled an identification of usage-dependent and usage-independent battery aging.

After five months of cycling, corresponding to a driven distance of 50,000 km, cell degradation has varied substantially with different operation conditions. Our paper provides valuable new insights on the impact of regenerative braking on battery aging: A higher level of regenerative braking has generally led to reduced battery aging. This can be attributed to a reduction of lithium plating, as the depth of discharge is reduced with an increased amount of charge recovered by regenerative braking. Our study has shown that it is not the short-time recharging with high current rates, but the long-lasting charging periods, even with only low current rates, that promotes lithium plating. Moreover, the comparison of usage-dependent and usage-independent battery aging has revealed that cyclic aging decreases with temperature, whereas calendar aging increases with temperature. Thus, battery life can be extended by optimized operating conditions.

In this paper, we provide advice for optimizing the operating conditions for Li-ion battery systems in electric vehicles. Not only regenerative braking, but also temperature and $\mathrm{SoC}$, is considered for optimal operating strategies maximizing battery life. Based on the results of our experimental study, achieving a driven distance of $100,000 \mathrm{~km}$ with only $10 \%$ capacity fade appears to be possible. Such a low battery aging is essential to promote the spread of electric vehicles, as it reduces the total cost of ownership, which is a prerequisite for the long-term success of electric vehicles.
\end{abstract}

Keywords: Li-ion battery, electric vehicle, aging, cycle life, regenerative braking 


\section{Introduction}

An electric vehicle can recover energy during braking by using its electric motor as an electric generator. This regenerative braking leads to a partial recharging of the vehicle's traction battery, thus, increasing range and efficiency. Braking actions in an automobile usually last only few seconds. Hence, the traction battery is repeatedly recharged by short charging periods. The magnitude of such a recharge period depends on the rate of deceleration. Even during 'moderate' braking, the battery has to cope with substantial current amplitudes. In today's electric vehicles, the traction battery is usually composed of Li-ion cells, which have strict operational voltage limitations. Exceeding these limitations intensifies aging and can lead to safety issues. Moreover, high charging currents can also damage the cells irreversibly, thus, reducing cycle life. Obtaining a long cycle life for the battery system is a prerequisite for the longterm success of electric vehicles. Hence, a profound knowledge of the determining factors for battery aging is essential. Therefore, we present an experimental cycle life study demonstrating the effects of regenerative braking on the aging of $\mathrm{Li}$ ion cells under different operating conditions.

\section{Fundamentals}

For the design of a meaningful cycle life study, the general limitations of charging Li-ion cells have been considered. Moreover, an appropriate cell has been selected and a representative load profile has been defined.

\subsection{Charging Li-Ion Cells}

The charging process of Li-ion cells is mainly limited by two factors: lithium plating on the anode and oxidation of the electrolyte solution due to high potentials at the cathode [1][2]. Both undesired side reactions lead to an irreversible loss of cyclable lithium. Moreover, they promote the growth of resistive surface layers. [1][3][4]

Lithium plating describes the reduction of $\mathrm{Li}^{+}$ ions, dissolved in the electrolyte, to metal lithium at the anode's surface, which takes place instead of the regular intercalation as neutral lithium atoms into the host lattice structure of the active material [5]. It can originate from limitations in charge transfer or lithium solid diffusion [6][7]. Lithium plating can occur, when the anode potential drops below the equilibrium potential of $\mathrm{Li}^{+} / \mathrm{Li}$ [6]. Some of the plated lithium later reacts irreversibly with the electrolyte and forms insoluble side products [3][4]. Graphite anodes, which are used in most Li-ion cells, are very prone to lithium plating due to their low equilibrium potential, especially at high states of charge (SoC) [8]. As a general trend, lithium plating increases with higher SoC, higher charging currents, and reduced temperature [6][8]. All in all, charging currents for graphite-based Li-ion cells are mainly limited by the intercalation kinetics at the anode [9].

The charging voltage is limited by the oxidation of electrolyte solvents, which occurs at high cathode (over)potentials [1][2]. Excessive overcharging can entail gas evolution, overpressure inside the cell, an opening of the cell's safety vent, and leakage of electrolyte. As the organic electrolytes of Li-ion batteries are highly flammable, this can lead to a fire or an explosion of the cell [10]. Thus, complying with the maximum cell voltage, specified by the manufacturer, is essential for safe operation and long cycle life.

Whereas a maximum value for the cell voltage is always defined in the datasheet, there are often only recommended current values for continuous charging. No detailed information is provided, which current rate can be applied to the cell for which period of time without generating plated lithium, which depends strongly on SoC and temperature. To investigate the impact of recharging the battery during regenerative braking on cycle life, an experimental study is presented in this paper.

\subsection{Cell Characterization}

In our cycle life study, commercial 18650 cells from a well-established manufacturer with mature production processes have been examined. Stable production processes guarantee reliable results without substantial variations from production. As electric vehicles have to provide a sufficient driving range, high-energy cells are typically used for their traction battery systems. For our cycle life study, highenergy Li-ion cells with NCA chemistry and a minimum nominal capacity of $2.8 \mathrm{Ah}$ have been selected (Panasonic NCR18650PD). These cells feature high specific energy of $220 \mathrm{Wh} / \mathrm{kg}$, high energy density of $570 \mathrm{Wh} / \mathrm{l}$, and low internal resistance of about $20 \mathrm{~m} \Omega$. Good capacity utilization at elevated discharge currents, up to $10 \mathrm{~A}$, and good discharge performance at low temperature, even below $0{ }^{\circ} \mathrm{C}$, also qualify the cells for electric vehicle applications. [11]

The aging of Li-ion batteries generally comprises usage-dependent and usage-independent degradation. Both lead to a loss of capacity and a rise of internal resistances. The end of life is often defined as a capacity loss of $20 \%$ or a resistance rise by $100 \%$. [12][13] 


\subsection{Vehicle Load Profiles}

To investigate the impact of regenerative braking on battery aging in an electric vehicle, an appropriate load profile has been determined. Moreover, it has been scaled down to cell level for our experimental cycle life study. [14]

As there are no specific load or driving profiles for electric vehicles, driving cycles for conventional vehicles have been used to derive load profiles for the traction battery of an electric vehicle.

The study by Neudorfer et al. has analyzed different driving cycles used as standardized references for fuel consumption measurements [15]. It is distinguished between 'modal' and 'stylistic' driving cycles. Modal cycles are artificial driving cycles that consist of several sections with constant velocity. In contrast, stylistic driving cycles represent realistic driving scenarios with frequently changing velocities. For our cycle life study, only stylistic driving cycles have been considered, as they produce a more realistic load scenario.

To generate a load profile for the battery of an electric vehicle, a simplified vehicle model has been used. This simulation model considers different driving resistances to compute the necessary driving power at each time step. Thus, the driving cycle's velocity profile is converted into a power profile. As described in [16], driving power $P_{\text {vehicle }}$ can be calculated as

$$
P_{\text {vehicle }}=F_{\text {req }} \cdot v=\left(F_{R}+F_{A}+F_{C}+F_{I}\right) \cdot v
$$

where $F_{\text {req }}$ represents the sum of all driving resistances and $v$ is the velocity of the vehicle. $F_{r e q}$ consists of the following simplified driving resistances:

- Rolling resistance: $F_{R}=\left(m_{V}+m_{L}\right) \cdot g \cdot f_{\text {roll }} \cdot \cos (\alpha)$

- Aerodynamic drag: $F_{A}=1 / 2 \cdot \rho_{A} \cdot c_{W} \cdot A_{A} \cdot\left(v+v_{A}\right)^{2}$

- Climbing resistance: $F_{A}=\left(m_{V}+m_{L}\right) \cdot g \cdot \sin (\alpha)$

- Acceleration resistance: $F_{I}=\left(e \cdot m_{V}+m_{L}\right) \cdot a$

where the acceleration $a$ is the derivative of the vehicle's velocity and $v+v_{A}$ is the relative velocity between air and vehicle. Table 1 provides a description of all symbols used and their values assumed for the load profile calculation. The values of vehicle A, a sub-compact urban vehicle for up to two passengers, are used in this study. Vehicle $\mathrm{B}$ represents a conventional compact car for four passengers.

To translate the driving power $P_{\text {vehicle }}$, required to accelerate and decelerate the vehicle, into a battery load $P_{\text {battery }}$, the drivetrain efficiency $\eta$ and the power consumption $P_{\text {aux }}$ of auxiliary consumers, such as lighting, heating, or driving assistance systems, have to be considered.
Table 1. Parameters for load profile computation

\begin{tabular}{|c|l|c|c|}
\hline Symbol & \multicolumn{1}{|c|}{ Description (Unit) } & $\begin{array}{c}\text { Vehicle } \\
\text { A }\end{array}$ & $\begin{array}{c}\text { Vehicle } \\
\text { B }\end{array}$ \\
\hline$m_{V}$ & Mass of vehicle $(\mathrm{kg})$ & 625 & 1350 \\
\hline$m_{L}$ & Mass of load $(\mathrm{kg})$ & 150 & 200 \\
\hline$g$ & Gravitational acceleration $\left(\mathrm{m} / \mathrm{s}^{2}\right)$ & 9.81 & 9.81 \\
\hline$f_{\text {roll }}$ & Rolling resistance coefficient $(-)$ & 0.007 & 0.011 \\
\hline$\alpha$ & Angle of inclination $\left(^{\circ}\right)$ & 0 & 0 \\
\hline$\rho_{A}$ & Density of air $\left(\mathrm{kg} / \mathrm{m}^{3}\right)$ & 1.2 & 1.2 \\
\hline$c_{W}$ & Drag coefficient $(-)$ & 0.22 & 0.30 \\
\hline$A_{A}$ & Cross-sectional area $\left(\mathrm{m}^{2}\right)$ & 1.69 & 2.20 \\
\hline$v_{A}$ & Air velocity $(\mathrm{m} / \mathrm{s})$ & 0 & 0 \\
\hline$e$ & Factor for rotational masses $(-)$ & 1.1 & 1.1 \\
\hline$\eta$ & Efficiency (-) & 0.75 & 0.80 \\
\hline$P_{\text {aux }}$ & Auxiliary power consumption $(\mathrm{W})$ & 500 & 1000 \\
\hline$N$ & Number of cells $(-)$ & 1296 & 2500 \\
\hline
\end{tabular}

Due to common sign conventions, positive values of driving power indicate acceleration, whereas positive battery currents represent a charging of the battery. The following two equations, depending on the direction of the power flow of the motor, account for these conventions:

- acceleration: $P_{\text {battery }}=-\left(P_{\text {vehicle }} / \eta+P_{\text {aux }}\right)$

- deceleration: $P_{\text {battery }}=-\left(P_{\text {vehicle }} \cdot \eta+P_{\text {aux }}\right)$

For simplicity reasons, a constant drivetrain efficiency of $\eta=0.75$ and a constant auxiliary power consumption of $P_{a u x}=500 \mathrm{~W}$ are assumed. These simplifications are tolerable, as for the investigation of battery aging, the qualitative distribution of charge and discharge loads is substantially more important than the precise quantitative values at each time step. All presented calculations do not have the aim to describe one specific electric vehicle as realistic and accurate as possible, but to generate a generic load profile suitable for the analysis of battery aging related to regenerative braking.

As a next step, the load profile is converted from the level of an entire traction battery to cell level by dividing the total power $P_{\text {battery }}$ by the number of cells $N$ in the battery pack:

$$
P_{\text {cell }}=P_{\text {battery }} / N
$$

This yields the power profile $P_{\text {cell }}$ for a single cell. In order to obtain a demanding load profile, a small battery pack of 1296 cylindrical 18650 cells with a total energy content of about $13 \mathrm{kWh}$ is assumed. This amount of energy is sufficient for the regarded sub-compact vehicle to provide a driving range of at least $100 \mathrm{~km}$.

Although the load profile has been computed for a sub-compact car, comparable results can also be obtained for a larger vehicle, which has, on the one hand, a higher mass and more engine power, but, on the other hand, a larger battery system. The parameter set of vehicle $\mathrm{B}$, listed in Table 1 , leads to a rather similar load profile as vehicle configuration A. 
Load profiles for battery aging experiments are usually defined as current profiles instead of power profiles. This guarantees the same charge throughput for each cell, independent of its SoC or terminal voltage. This leads to a better comparability of the results. Therefore, the power profile is divided by a reference voltage $U_{\text {ref }}$ to obtain a current profile:

$$
I_{\text {cell }}=P_{\text {cell }} / U_{\text {ref }}
$$

Since the investigations within this paper cover a wide $\mathrm{SoC}$ range, $U_{\text {ref }}$ has been set to $3.6 \mathrm{~V}$, which is the nominal cell voltage, specified by the manufacturer.

For different European and American driving cycles, velocity profiles from [17] have been transformed into current profiles for a single Li-ion cell with a numerical fixed-step solver and a step size of $1 \mathrm{~s}$ (see Table 2). Fig. 1 depicts the current histograms of several driving cycles, representing different driving conditions. This serves as a basis for the design of the cycle life study.

\section{Experimental Aging Study}

In this section, the design of the cycle life experiment is presented. This comprises the selection of an appropriate load profile, the definition of the variables for parameter variations, the tracking of cell aging, and the overall cycling process. Additional investigations on calendar life complete our aging study.
Table 2. Comparision of eight European and American driving cycles including the maximum amount of charge recovered in the driving simulation with vehicle $A$.

\begin{tabular}{|l|c|c|c|c|}
\hline & $\begin{array}{c}\text { Duration } \\
\text { (min) }\end{array}$ & $\begin{array}{c}\text { Average } \\
\text { Velocity } \\
\text { (km/h) }\end{array}$ & $\begin{array}{c}\text { Discharged } \\
\text { Ampere- } \\
\text { hours (Ah) }\end{array}$ & $\begin{array}{c}\text { Maximum } \\
\text { Charge } \\
\text { Recovery }\end{array}$ \\
\hline ARTEMIS Urban & 16,5 & 16,2 & 0,151 & $25 \%$ \\
\hline ARTEMIS Road & 18,0 & 57,5 & 0,354 & $17 \%$ \\
\hline $\begin{array}{l}\text { ARTEMIS } \\
\text { Motorway 130 }\end{array}$ & 17,8 & 96,9 & 0,773 & $8 \%$ \\
\hline NYCC & 10,0 & 11,4 & 0,063 & $21 \%$ \\
\hline FTP72 & 22,8 & 31,5 & 0,244 & $19 \%$ \\
\hline LA92 & 23,9 & 39,7 & 0,401 & $20 \%$ \\
\hline HWFET & 12,8 & 77,7 & 0,277 & $6 \%$ \\
\hline US06 & 10,0 & 77,3 & 0,369 & $15 \%$ \\
\hline
\end{tabular}

\subsection{Load Profile}

For investigating the impact of regenerative braking on battery aging, highway driving cycles are beneficial, as they feature a higher energy consumption than urban and rural driving cycles. This leads to a higher charge throughput and is, consequently, simulating a higher driving distance per time (see Table 2). Moreover, this represents a worst-case usage scenario, as electric vehicles are often used in urban traffic conditions, where, despite of more frequent stop-and-go driving conditions, the battery load is considerably lower.

Table 2 also shows that the amount of energy that can be recovered by regenerative braking in highway driving cycles amounts to $8 \%$ for the Artemis Motorway 130, $6 \%$ for the HWFET, and $15 \%$ for the US06 driving cycle. Since the American US06

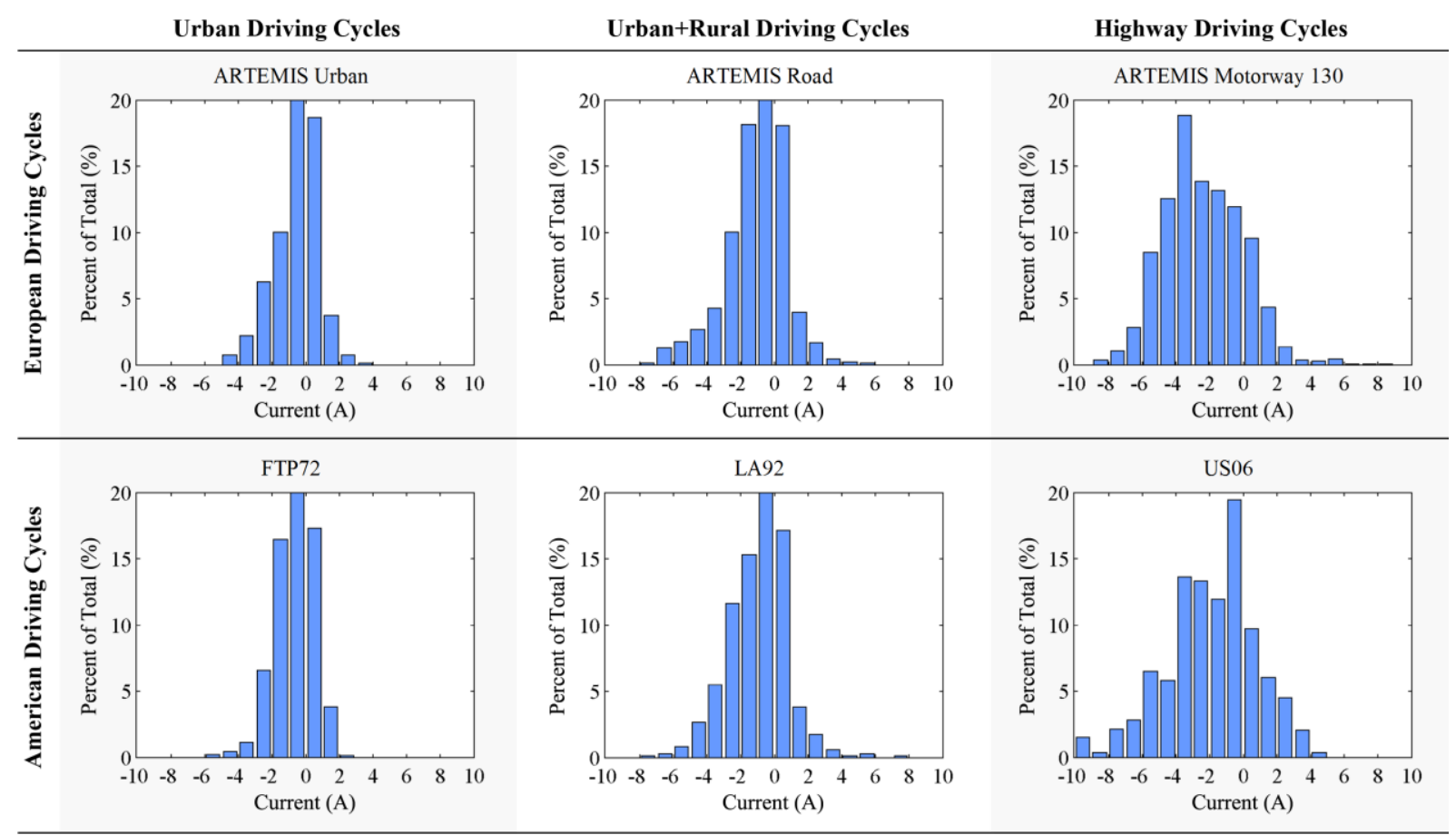

Fig. 1. Distribution of cell currents for different European and American driving cycles as percent of total driving cycle duration. The parameters of vehicle configuration A, used in this driving simulation, are listed in Table 1. 
driving cycle recovers substantially more charge than the other two highway driving cycles and since it contains a considerable percentage of regenerative braking events with a current magnitude above $2 \mathrm{~A}$ (see Fig. 1), it has been selected for our cycle life study. The velocity profile of this highway driving cycle is illustrated in Fig. 2.

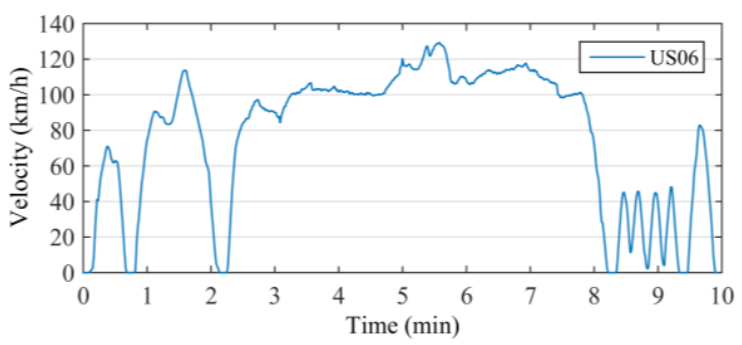

Fig. 2. Velocity profile of US06 highway driving cycle

\subsection{Parameter Variations}

In our cycle life study, different influencing factors are investigated: Temperature, SoC, and the magnitude of regenerative braking have been varied. For each factor, at least three values have been tested. Different magnitudes of regenerative braking have been implemented by varying the maximum recharge current rate of the load profile.

\subsubsection{Temperature}

Three temperatures have been investigated: $25^{\circ} \mathrm{C}$ is considered as a standard operating temperature for the Li-ion cells. To cover a realistic spectrum of average operating temperatures for an electric vehicle, an additional high and low temperature level of $40^{\circ} \mathrm{C}$ and $10^{\circ} \mathrm{C}$ has been examined. This can be interpreted as summer and winter conditions, when the battery pack cannot be cooled down or heated up to $25^{\circ} \mathrm{C}$. Three thermal chambers have been used to establish the different environmental conditions.

\subsubsection{Magnitude of Regenerative Braking}

The cycle life study is conducted on a BaSyTec CTS battery test system with 32 independent $5 \mathrm{~A}$ test channels. Within the computed US06 load profile, charge currents reach up to $4.5 \mathrm{~A}$. Thus, all recharge magnitudes can be covered by the battery test system. High discharge currents, however, have to be truncated at $-5.5 \mathrm{~A}$, which is the limit of the test system. As variations in recharging during periods of regenerative braking are the center of interest, the limitations in discharge direction are negligible for this cycle life study.

To analyze the impact of regenerative braking on battery aging, four different levels of recharge cur- rents have been defined: The first level is 'no recharging' (no $I_{r e}$ ), which corresponds to no regenerative braking at all. The second level limits recharge currents $I_{r e}$ to $1 \mathrm{~A}\left(I_{r e} \leq 1 \mathrm{~A}\right)$ and is able to recover $8 \%$ of the ampere-hours discharged during the driving cycle. This correlates with approximately half of the maximum recoverable amount of charge of $15 \%$. The third level of regenerative braking has been defined as $I_{r e} \leq 2 \mathrm{~A}$, covering more than $80 \%$ of the maximum recoverable amount of charge. The fourth level, covering all recharge pulses, is labeled as $I_{r e} \leq 4 \mathrm{~A}$. Although there are two short charge pulses of $4.5 \mathrm{~A}$, this designation is chosen, as less than $0.5 \%$ of the recovered amount of charge is generated by these two current peaks. Fig. 3 illustrates the different current profiles, representing the four magnitudes of regenerative braking examined.

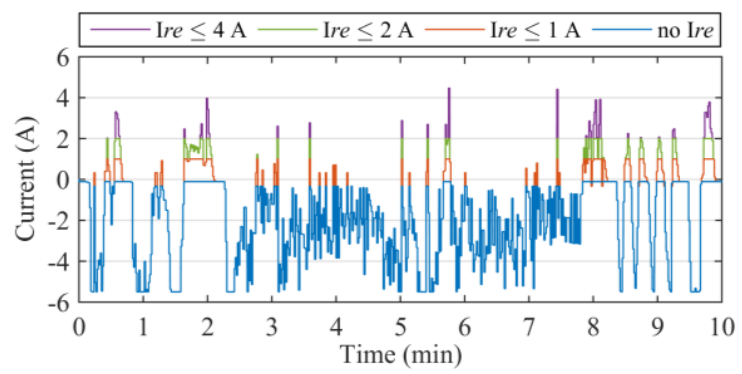

Fig. 3. US06 load profiles for a single Li-ion cell with different magnitudes of regenerative braking, represented by different recharge current $\left(I_{r e}\right)$ limitations

\subsubsection{State of Charge Levels}

One run of the US06 driving cycle corresponds to a driven distance of $13 \mathrm{~km}$. To obtain a more representative driving distance for the cycle life study, two runs of the load profile are performed in series before charging the cell again. As the total range of the considered electric vehicle is at least $100 \mathrm{~km}$, approximately one fourth of the battery's capacity is depleted by two consecutive runs of the highway driving cycle.

Within our cycle life experiment, three SoC levels are examined. The SoC levels are defined by the maximum charging voltage for a constant-current (CC) charging procedure with a charging current of $0.7 \mathrm{~A}(=0.25 \mathrm{C})$. As shown in Fig. 4, the charging voltages for 'low SoC', 'medium SoC' and 'high SoC' are $3.7 \mathrm{~V}, 3.9 \mathrm{~V}$, and $4.1 \mathrm{~V}$, respectively. Fig. 4 also illustrates the cycling windows for the three SoC levels. The remaining SoC safety margins prevent overcharging during recharge pulses at high SoC and allow to perform the cycling procedure also on aged cells approaching the end-of-life criterion of $20 \%$ capacity loss.

A slight correction of charging voltages has been conducted for the cells at $10^{\circ} \mathrm{C}$ : As higher internal 


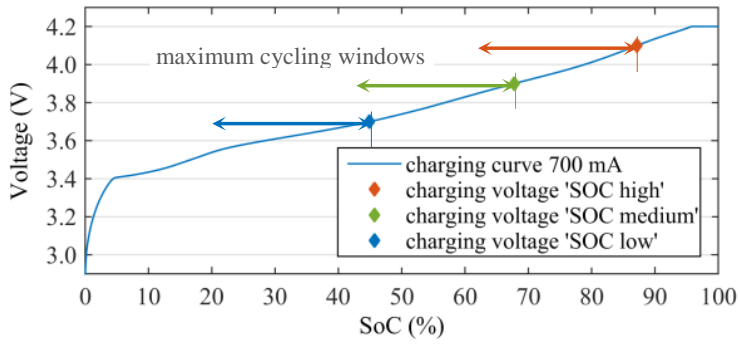

Fig. 4. Voltage curve for constant-current-constantvoltage charging at $25^{\circ} \mathrm{C}$. The double arrows illustrate the cycling windows, when there is no regenerative braking.

resistances at low temperatures lead to higher terminal voltages during charging, the low SoC level has been set to $3.75 \mathrm{~V}$ and the medium SoC has been set to $3.925 \mathrm{~V}$. The high SoC level has remained at $4.1 \mathrm{~V}$ in order not to expose the cell to higher voltage potentials, which might have increased lithium plating or electrolyte decomposition.

\subsection{Cycle Life Test Procedure}

To track the aging of the Li-ion cells, a checkup routine has been defined, which consists of capacity and cell resistance measurements. This checkup routine is identical for all cells: At $25^{\circ} \mathrm{C}$, the cells are fully charged with a constant current of $0.7 \mathrm{~A}$ and a constant voltage of $4.2 \mathrm{~V}$, until the current drops below $0.1 \mathrm{~A}$. After that, the cells are discharged with a constant current of $3 \mathrm{~A}$ to $2.5 \mathrm{~V}$, followed by a constant voltage period with a cutoff current of $0.1 \mathrm{~A}$. The additional constant-voltage discharge period enables a more precise measurement of the cell's actual capacity.

All cells have performed a checkup at the beginning of the cycle life experiment and after each cycling sequence. The cycling sequence has been defined as follows: After placing the cell in its temperature chamber, it is charged with a constant current of $0.7 \mathrm{~A}(=0.25 \mathrm{C})$, until it reaches its dedicated charging voltage. After a pause of $5 \mathrm{~min}$, two consecutive driving cycle runs are performed with a pause of $1 \mathrm{~min}$ after each run. This combination of constant-current charging and two driving cycle runs is repeated 400 times. As each of the 400 cycles depletes about one fourth of the cell's capacity, each cell has been charged and discharged approximately 100 times its nominal capacity $(=280 \mathrm{Ah})$ between two checkups.

As the Li-ion cells investigated in this aging study exhibit considerably higher changes in capacity than in internal resistance, this paper focuses on the examination of capacity changes.

\subsection{Calendar Life}

In order to separate usage-dependent and usage-independent aging, a calendar life study has been conducted in parallel: Additional cells have been stored at various SoC and different temperatures. Fig. 5 shows the eight SoC levels, ranging from $0 \%$ to $100 \%$ SoC. The storage temperatures are identical to the cycling temperatures: $10^{\circ} \mathrm{C}, 25^{\circ} \mathrm{C}$, and $40^{\circ} \mathrm{C}$. All stored cells are examined periodically at $25^{\circ} \mathrm{C}$ with the same checkup routine as the cycled cells. After each checkup, the cells are charged to their storage SoC and placed in the thermal chambers again.

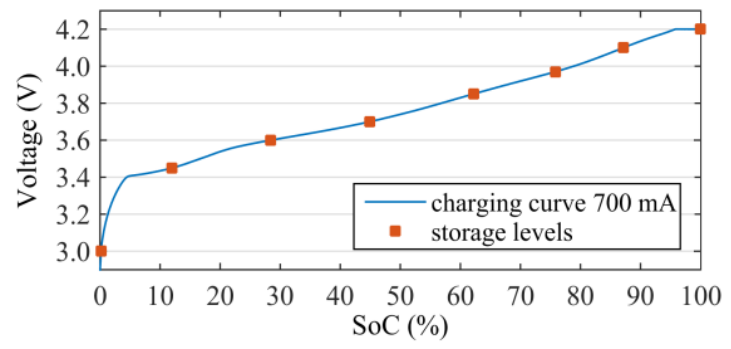

Fig. 5. SoC levels examined in the calendar life study

\section{Results and Discussion}

After five months of cycle life testing, the Li-ion cells have been exposed to a charge throughput of $1,400 \mathrm{Ah}$, which corresponds to 500 nominal full cycles and a driven distance of approximately $50,000 \mathrm{~km}$. Variations in cell aging can be observed for the different cycling conditions. In the following sections, these results are presented and discussed together with the results from the calendar life study. Finally, optimized operational strategies are derived from the findings.

\subsection{Calendar Life}

The SoC-dependent calendar aging has been revealed by the stored Li-ion cells. Fig. 6 illustrates the capacity fade after about five months of storage for the eight different SoC and the three storage temperatures.

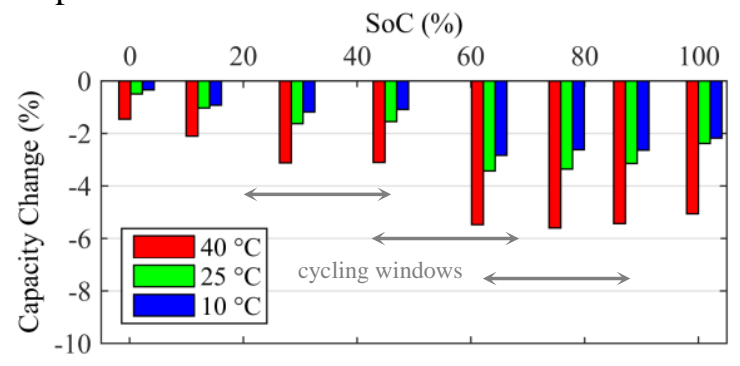

Fig. 6. SoC-dependent capacity fade after five months of storage. The double arrows illustrate the three operating windows at low, medium, and high SoC. 
As expected, cell aging accelerates with temperature: The capacity loss at $40^{\circ} \mathrm{C}$, ranging between $2 \%$ and $6 \%$, is almost twice as high as at $25^{\circ} \mathrm{C}$. A further lowering of the temperature, however, does not decelerate aging considerably. At $10^{\circ} \mathrm{C}$, there is still a capacity loss between $0.5 \%$ and $3 \%$, depending on SoC.

Fig. 6 also shows that cells stored in a discharged state below $20 \%$ SoC exhibit the least capacity fade. Storage levels between $20 \%$ and $50 \%$ SoC cause a medium degradation rate. The fastest capacity fade occurs at a SoC interval between $60 \%$ and $90 \%$. A fully charged cell, however, shows a somewhat slower capacity fade again.

The data from the calendar life study serve as a baseline for the following investigations on cycle life under different operating conditions. For the three cycling windows, depicted in Fig. 6, mean curves for calendar aging have been computed based on the capacity changes of the cells contained in the respective window. For the comparison of calendar and cyclic aging, capacity curves from calendar aging are scaled accordingly to the about four weeks of cycling between two consecutive checkups in the cycle life study.

\subsection{Cycle Life}

The main focus of this paper is on investigating the impact of regenerative braking on battery aging. Fig. 7 shows the results of the experimental cycle life study: Capacity losses are depicted separately for the three temperatures and the three SoC levels. In each subplot, the different magnitudes of regenerative braking are compared. Moreover, calendar aging is included to distinguish between usage-dependent and usage-independent capacity losses.

\subsubsection{Capacity Fade at $40{ }^{\circ} \mathrm{C}$}

At $40^{\circ} \mathrm{C}$, the Li-ion cells cycled within the low and medium $\mathrm{SoC}$ window show almost no dependency on the level of regenerative braking. All curves lie closely together. At the end of the cycling process, cells at low SoC exhibit a capacity loss of almost $6 \%$ and cells at medium SoC of almost $7 \%$. The additional capacity loss at medium $\mathrm{SoC}$ can be attributed to increased calendar aging. Thus, the capacity loss supplementary to calendar aging due to cycling is considered identical for both $\mathrm{SoC}$ levels and accounts for about $2.5 \%$ after the total charge throughput of $1,400 \mathrm{Ah}$.
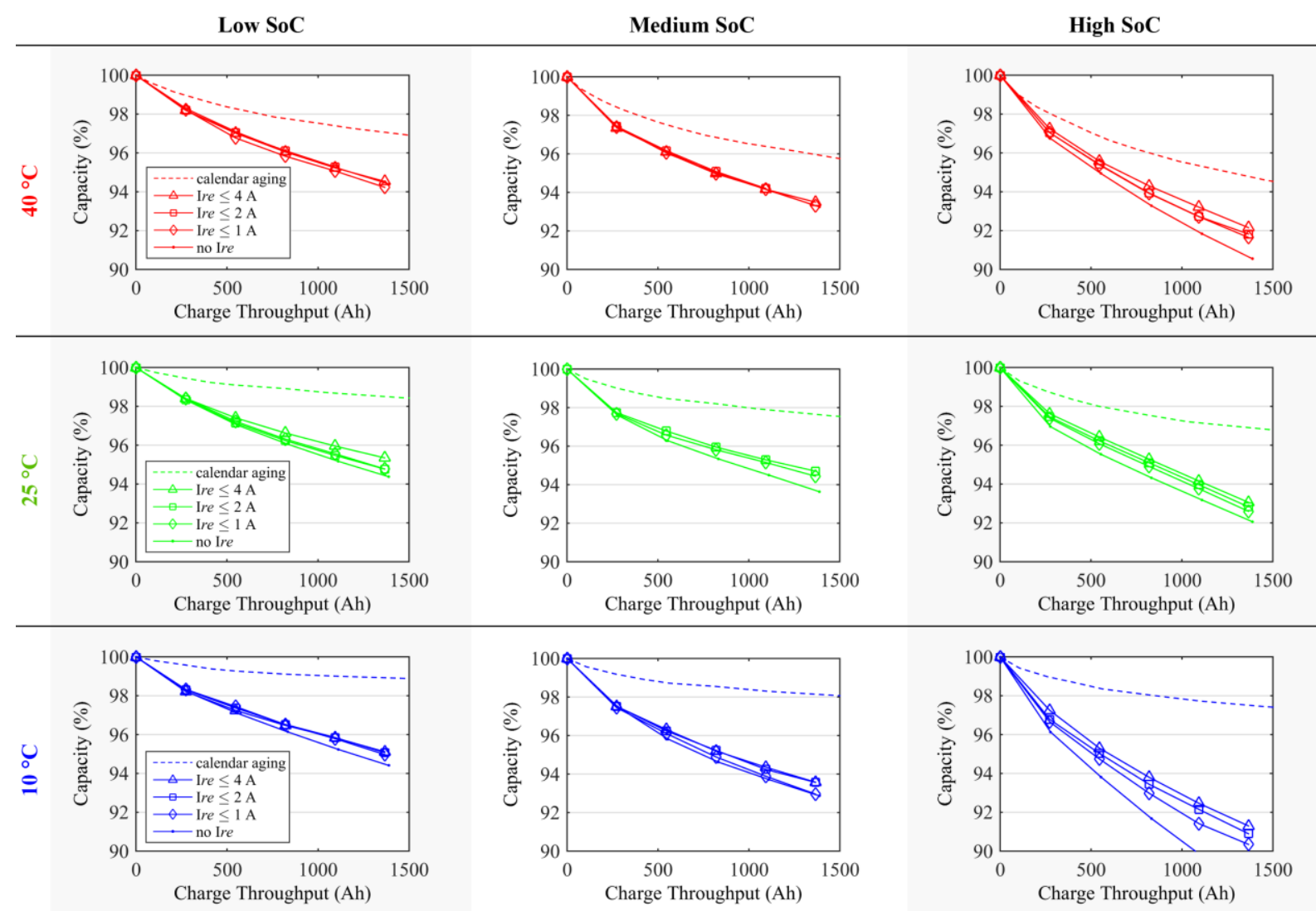

Fig. 7. Cell degradation during the cycle life experiment for the four magnitudes of regenerative braking at different temperatures and SoC. The checkup measurements have always been performed after a total charge throughput of about $280 \mathrm{Ah}$ (=280 Ah charged $+280 \mathrm{Ah}$ discharged), which represents a driven distance of $10,000 \mathrm{~km}$. The calendar aging curves are scaled accordingly to the about four weeks of cycling between two consecutive checkups. 
Regarding the high SoC level at $40{ }^{\circ} \mathrm{C}$, Fig. 7 demonstrates a dependency on regenerative braking, as the capacity curves diverge. A trend becomes apparent: A higher level of regenerative braking reduces the capacity loss. At the end of the test, the capacity loss supplementary to calendar aging is about $2.5 \%$ for the cell with the maximum level of regenerative braking and more than $4 \%$ for the cell with no regenerative braking.

\subsubsection{Capacity Fade at $25^{\circ} \mathrm{C}$}

Unlike at $40^{\circ} \mathrm{C}$, Fig. 7 shows a dependency of battery aging on the level of regenerative braking for all three SoC levels at $25^{\circ} \mathrm{C}$ : Cells with a higher level of regenerative braking exhibit lower capacity losses, which corresponds to a longer cycle life. In analogy to $40{ }^{\circ} \mathrm{C}$, capacity fade increases with higher SoC level. At low and medium SoC, capacity losses due to cycling are comparable for the same levels of regenerative braking. At high SoC, intensified aging can be observed again: $\mathrm{Ca}-$ pacity losses due to cycling are more than 1 percentage point higher than at medium and low SoC. Compared to $40^{\circ} \mathrm{C}$, calendar aging is about 2 percentage points lower and cyclic aging is about 1 percentage point higher at $25^{\circ} \mathrm{C}$.

\subsubsection{Capacity Fade at $10{ }^{\circ} \mathrm{C}$}

At $10^{\circ} \mathrm{C}$, Fig. 7 presents again a dependency on the level of regenerative braking for all SoC levels. The most pronounced impact can be observed at the high SoC level, where capacity curves diverge markedly. Although calendar aging is low at $10^{\circ} \mathrm{C}$, the cells exhibit substantial capacity losses. After the charge throughput of 1,400 Ah, capacity losses due to calendar aging amount to $1 \%$ for low SoC, $2 \%$ for medium SoC, and $2.5 \%$ for high SoC. For the low and medium SoC level, additional losses of 4-5\% can be attributed to cyclic aging. At the high SoC level, the cell with maximum regenerative braking exhibits $6 \%$ of cyclic aging; for the cell with no regenerative braking, elevated cyclic losses of $10 \%$ are observed, which represents the most severe aging of all operating conditions. It can be assumed that the reduced kinetics at low temperature increase inhomogeneity inside the cell and that an uneven potential distribution intensifies aging effects.

\subsection{Reduced Aging due to Regenera- tive Braking}

Analyzing the capacity fade with respect to regenerative braking at different temperatures has re- vealed a common trend: A higher level of regenerative braking usually reduces battery aging. This trend can be observed most clearly for high SoC and low temperature. These operating conditions are known to aggravate lithium plating. Thus, the reduced aging is considered as a reduction of lithium plating. Hence, the concern of regenerative braking generating more lithium plating has been disproved for all operating conditions examined in this cycle life study. Moreover, our results lead to the conclusion that regenerative braking helps to reduce lithium plating.

This can be explained by a decreased depth of discharge after the driving cycles: The more charge recovered by regenerative braking, the higher the SoC remains at the end of the driving cycle. Thus, the subsequent constant-current (CC) charging period becomes shorter. As lithium plating is reduced, when the $\mathrm{CC}$ charging periods become shorter, the $\mathrm{CC}$ charging periods appear as the main driver of lithium plating. Consequently, it is not the short recharging with high current rates during regenerative braking that promotes lithium plating, but the longlasting charging periods, when the vehicle is recharged by the grid, although the current rate is considerably lower.

To verify this assertion, capacity degradation has been plotted against the cumulated amount of charge transferred solely in the CC charging periods. Fig. 8 shows that, at the end of the cycle life experiment, the total ampere-hours charged during the CC periods are considerably lower for cells with higher levels of regenerative braking. Cells with maximum regenerative braking have only been charged with 1,200 Ah between the driving cycles, whereas cells without regenerative braking have been charged with the total 1,400 Ah during the CC charging periods. Comparing Fig. 7 and Fig. 8, the adjustments due to the different scale basis become obvious: At $10^{\circ} \mathrm{C}$ and $25^{\circ} \mathrm{C}$, capacity curves lie closer together in Fig. 8 for all SoC levels. At $40^{\circ} \mathrm{C}$, this effect can also be observed at high SoC. This confirms the direct correlation between cell degradation and the amount of charge transferred during the $\mathrm{CC}$ charging periods under most operating conditions.

Only for low and medium SoC at $40^{\circ} \mathrm{C}$, the correlation between cell degradation and the total charge throughput is in better agreement. Under these operating conditions, lithium plating is no determining factor for cell aging. As Fig. 7 shows, even under these operating conditions, regenerative braking does not intensify battery aging.

Thus, a high level of regenerative braking is beneficial for the cycle life of a Li-ion battery system in 


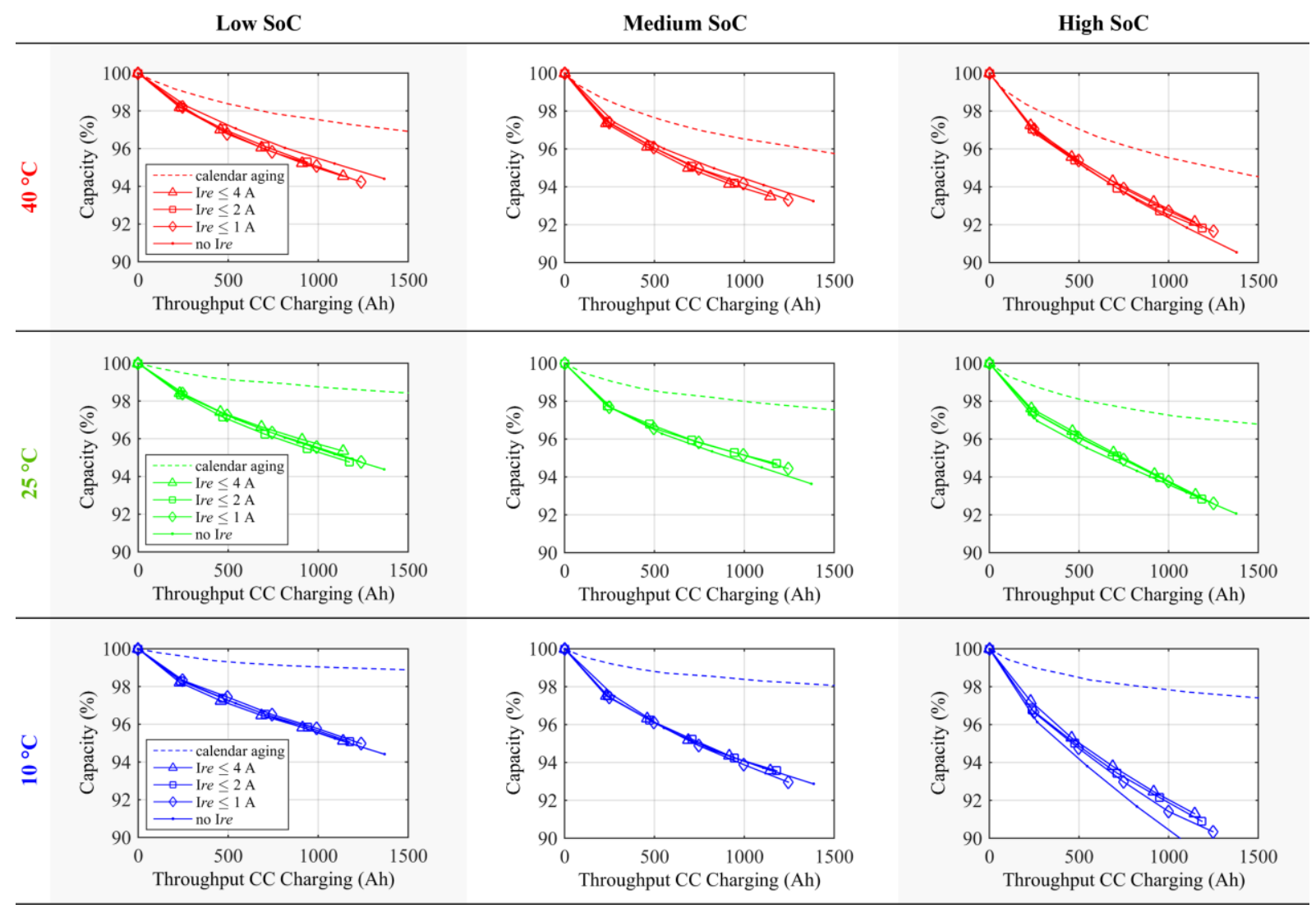

Fig. 8. Cell degradation against cumulated charge throughput during constant-current (CC) charging periods. The same magnitudes of regenerative braking, temperatures, and SoC levels are presented as in Fig. 7. The scaling of the calendar aging curves is identical to Fig. 7.

an electric vehicle. By reducing the depth of discharge and the duration of the subsequent charging period, regenerative braking can reduce lithium plating considerably.

\subsection{Impact of Temperature}

Our aging study also demonstrates that the contributions of calendar aging and cyclic aging vary with temperature. Fig. 9 compares calendar aging with the aging results of one level of regenerative braking for all three temperatures. Calendar aging increases substantially with elevated temperature: After five months at $40^{\circ} \mathrm{C}$, the capacity fade is about 2 percentage points higher than at $25^{\circ} \mathrm{C}$ and about 2.5 percentage points higher than at $10^{\circ} \mathrm{C}$. In contrast, cyclic aging decreases with temperature. At low SoC, cyclic aging at $40^{\circ} \mathrm{C}$ is approximately 1 percentage point lower than at $25^{\circ} \mathrm{C}$ and $10^{\circ} \mathrm{C}$. Cyclic aging at medium $\mathrm{SoC}$ is comparable to low SoC for $40^{\circ} \mathrm{C}$ and $25^{\circ} \mathrm{C}$, whereas it increases by 1 percentage point at $10^{\circ} \mathrm{C}$. At high SoC, lower temperatures exhibit intensified aging. Fig. 9 illustrates that cyclic aging has increased by about 1 percentage point at $25^{\circ} \mathrm{C}$ and by about 2 percentage points at $10^{\circ} \mathrm{C}$ compared to medium SoC.

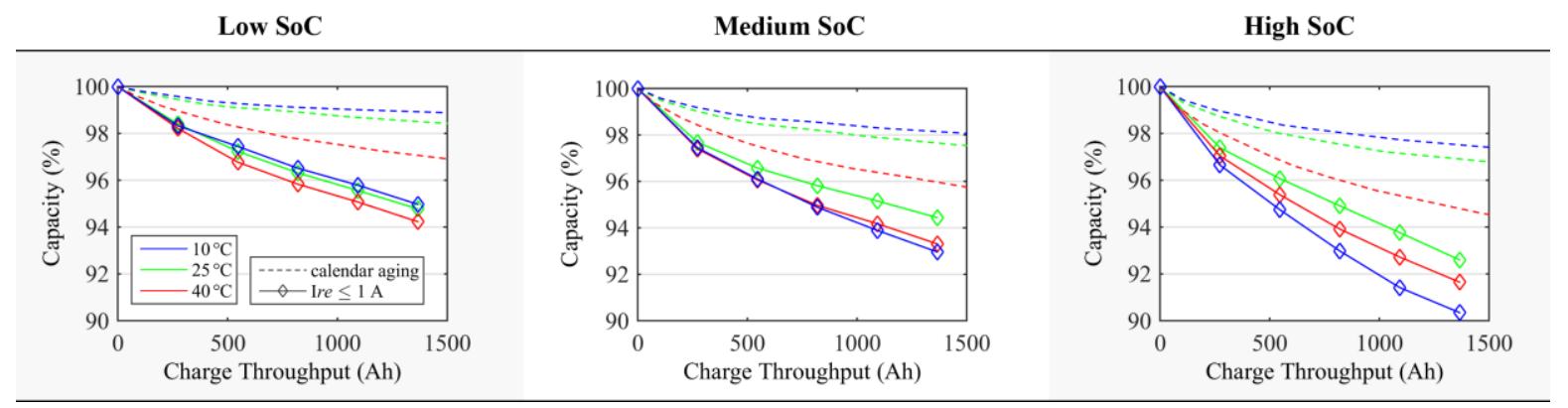

Fig. 9. Comparison of calendar aging and cyclic aging for the three temperatures investigated. The scaling of the calendar aging curves is identical to Fig. 7. 


\subsection{Optimal Operating Conditions}

Our study shows that cyclic aging increases with lower temperature and calendar aging increases with higher temperature. Thus, an optimization is necessary to minimize the aging of a Li-ion battery. During storage periods, temperature should be kept low to reduce calendar aging. When cycling the battery, especially when charging the battery, a higher temperature should be established to minimize aging due to lithium plating. When charging the battery for a longer time at low temperature, current rates should be kept low to reduce lithium plating.

In addition to temperature, the SoC level also influences aging substantially. High SoC levels have been detrimental for calendar and cyclic life. Thus, to minimize battery aging, a high SoC should be avoided whenever possible.

Moreover, long-lasting charging periods have been identified as main driver of lithium plating. Thus, reducing the depth of discharge during cycling can decrease battery aging. A high level of regenerative braking should be implemented, as it reduces the depth of discharge and has shown no detrimental effects on cycle life.

In our study, cells cycled at $25^{\circ} \mathrm{C}$ provide a good compromise between calendar and cyclic aging. Extrapolating their capacity degradation for maximum regenerative braking at low or medium SoC predicts a capacity loss of only about $10 \%$ after a driven distance of $100,000 \mathrm{~km}$. This highlights the potential of optimized operating conditions.

\section{Conclusion}

Our cycle life study has provided valuable new insights on the impact of regenerative braking on the aging of a Li-ion traction battery in an electric vehicle: Under all operating conditions investigated, there have been no detrimental effects of regenerative braking on battery life. Moreover, regenerative braking has prolonged cycle life by reducing the depth of discharge.

Our study has shown that it is not the short-time recharging with high current rates, but the longlasting charging periods, even with comparatively low amperage, that leads to battery aging related to lithium plating. By reducing the depth of discharge and the duration of the subsequent recharging period, regenerative braking has decreased lithium plating. This has been observed most pronouncedly at high SoC and low temperature. These conditions are known as the main drivers of lithium plating.
Although our study provides clear insights, there are still open questions for further research on battery aging related to regenerative braking, such as determining the maximum current rates applicable without generating plated lithium, the interdependencies of cycle depth and aging, and the aging behavior below $10^{\circ} \mathrm{C}$.

Our additional investigations on calendar aging have revealed that cyclic aging decreases with temperature, whereas calendar aging increases. Moreover, the results of the calendar life study have exhibited an accelerated cell degradation at a SoC above $60 \%$. Thus, an optimization of operating conditions is essential to maximize battery life.

First cycle life projections, based on an extrapolation of the experimental data, show that under optimized operational conditions, there is only a capacity fade of about $10 \%$ after a driven distance of $100,000 \mathrm{~km}$. With such low degradation, battery replacements become redundant during the life of most electric vehicles. Thus, optimized operational strategies with a high magnitude of regenerative braking can promote the spread of electric vehicles by reducing the total cost of ownership.

\section{Acknowledgments}

The authors would like to thank the German Federal Ministry of Education and Research for their financial support. [FKZ: 16N12101]

\section{References}

[1] S. S. Zhang, The effect of the charging protocol on the cycle life of a Li-ion battery, Journal of Power Sources, ISSN 0378-7753, 161 (2006), 1385-1391.

[2] P. E. de Jongh, et al., Effect of current pulses on lithium intercalation batteries, Solid State Ionics, ISSN 0167-2738, 148 (2002), 259-268.

[3] P. Arora, et al., Capacity Fade Mechanisms and Side Reactions in Lithium-Ion Batteries, Journal of the Electrochemical Society, ISSN 1945-7111, 145 (1999), pp. 3543-3553.

[4] Z. Li, et al., A review of lithium deposition in lithium-ion and lithium metal secondary batteries, Journal of Power Sources, ISSN 0378-7753, 254 (2014), 168-182.

[5] V. Zinth, et al., Lithium plating in lithium-ion batteries at sub-ambient temperatures investigated by in situ neutron diffraction, Journal of Power Sources, ISSN 0378-7753, 271 (2014), 152-159.

[6] N. Legrand, et al., Physical characterization of the charging process of a Li-ion battery and prediction of Li plating by electrochemical modelling, Journal 
of Power Sources, ISSN 0378-7753, 245 (2014), 208-216.

[7] B. K. Purushothaman, et al., Rapid Charging of Lithium-Ion Batteries Using Pulsed Currents, Journal of the Electrochemical Society, ISSN 1945-7111, 153 (2006), A533-A542.

[8] S. S. Zhang, et al., Study of the charging process of a LiCoO2-based Li-ion battery, Journal of Power Sources, ISSN 0378-7753, 160 (2006), 1349-1354.

[9] Y. Yamada, et al., A superconcentrated ether electrolyte for fast-charging Li-ion batteries, Chemical Communications, ISSN 1359-7345, 49 (2013), 11194-11196.

[10] M. Brand, et al., Electrical safety of commercial Li-ion cells based on NMC and NCA technology compared to LFP technology, 2013 World Electric Vehicle Symposium and Exhibition (EVS27).

[11] Panasonic, Introduction of NCR18650PD

[12] E. Sarasketa-Zabala, et al., Calendar ageing analysis of a LiFePO4/graphite cell with dynamic model validations: Towards realistic lifetime predictions, Journal of Power Sources, ISSN 0378-7753, 272 (2014), 45-57.

[13] R. Spotnitz, Simulation of capacity fade in lithium-ion batteries, Journal of Power Sources, ISSN 0378-7753, 113 (2003), 72-80.

[14] P. Keil, et al., Influence of Regenerative Braking on the Aging of the Li-Ion Battery of an Electric Vehicle, Conference on Future Automotive Technology, Garching, Germany, 2014.

[15] H. Neudorfer, et al., Analyse von unterschiedlichen Fahrzyklen für den Einsatz von Elektrofahrzeugen, Elektrotechnik \& Informationstechnik, ISSN 0932-383X, 123 (2006), 352-360.

[16] B. Heißing, et al., Chassis Handbook: Fundamentals, Driving Dynamics, Components, Mechatronics, Perspectives, ISBN 3834809942, Springer Fachmedien Wiesbaden, Vieweg + Teubner Verlag, 2010.

[17] www.dieselnet.com/standards/cycles

\section{Authors}

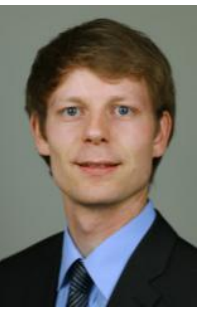

Dipl.-Ing. Peter Keil earned his diploma degree in mechanical engineering from the Technische Universität München in 2010. Currently, he is working as a research associate at the Institute for Electrical Energy Storage Technology at the Technische Universität München. His main research activities are in the fields of electrical and thermal characterization of Li-ion cells for investigating cell aging.

Prof. Dr.-Ing. Andreas Jossen holds a professorship at the Technische Universität München and he is the founder and head of the Institute of Electrical Energy Storage Technology. His research activities are modeling, simulation, and characterization of rechargeable batteries and fundamental and applied topics in battery systems, such as battery topologies, state determination, and control of battery systems. 